\title{
Probing variation of the fine-structure constant in runaway dilaton models using Strong Gravitational Lensing and Type Ia Supernovae
}

\author{
L. R. Colaço ${ }^{1, a}$, R. F. L. Holanda ${ }^{1, b}$, R. Silva $a^{1,2, c}$ \\ ${ }^{1}$ Departamento de Física Teórica e Experimental, Universidade Federal do Rio Grande do Norte, Natal, RN 59300-000, Brazil \\ ${ }^{2}$ Departamento de Física, Universidade do Estado do Rio Grande do Norte, Mossoró, RN 59610-210, Brazil
}

Received: 14 April 2021 / Accepted: 7 September 2021 / Published online: 16 September 2021

(C) The Author(s) 2021

\begin{abstract}
In order to probe a possible time variation of the fine-structure constant $(\alpha)$, we propose a new method based on Strong Gravitational Lensing and Type Ia Supernovae observations. By considering a class of runaway dilaton models, where $\frac{\Delta \alpha}{\alpha}=-\gamma \ln (1+z)$, we obtain constraints on $\frac{\Delta \alpha}{\alpha}$ at the level $\gamma \sim 10^{-2}$ ( $\gamma$ captures the physical properties of the model). Since the data set covers the redshift range $0.075 \leq z \leq 2.2649$, the constraints derived here provide independent bounds on a possible time variation of $\alpha$ at low, intermediate and high redshifts.
\end{abstract}

\section{Introduction}

The hypothesis of large numbers (HLN), proposed a long time ago by Paul Dirac [1], has opened many possible approaches associated with a variation of the constants of nature. For example, an early investigation addressed a possible variation of the gravitational constant $(G)$, but as the main result, this temporal dependence of $G$ was ruled out by [2] years after. Recently, the HLN has gained a lot of attention with the experimental advance. In this concern, Dirac's hypothesis has been tested in many physical contexts, e.g., by using geological evidence, no variation in $G$ was either found by investigating the effects on the evolution and asteroseismology of the low-mass star KIC 7970740 [3]. Considering the Earth-Moon system, competitive experiments have provided an $G$ upper bound, such as $\dot{G} / G=0.2 \pm 0.7 \times 10^{-12}$ per year [4]. From the string theory and other theories of modified gravity standpoint, on the other hand, $G$ assumes a variable gravitational constant $[5,6]$. Moreover, due to the

\footnotetext{
a e-mail: colacolrc@gmail.com (corresponding author)

b e-mail: holandarfl@gmail.com

c e-mail: raimundosilva@ fisica.ufrn.br
}

possibility of dynamical constants existing, some theories based on extra dimensions have also been discussed [7-9]. It is important to stress that General Relativity discards a fundamental dynamical constant due to possible violation of the Equivalence Principle [10].

Some observational measurements have also been considered to investigate a possible variation of the fine-structure constant (in electrostatic cgs units $\alpha=e^{2} / \hbar c$, where $e$ is the elementary charge, $\hbar$ the reduced Planck's constant, and $c$ the speed of the light). The absorption spectra of quasars, for instance, have been much used to explore a possible cosmological time variation of $\alpha$ [11-17], and also by the rare-earth element abundance data from Oklo [18]. Very recently, from 4 quasars spectral observations up to $z \approx 7.1$, no evidence for a temporal change has been found. However, when combining the four new measurements with a large existing sample of lower redshift measurements, a possible spatial variation was marginally preferred over a no-variation model [19]. By using the physics of the cosmic microwave background (CMB), some researchers have used CMB anisotropies measurements to test models with varying $\alpha$. For example, from the Planck satellite data [20,21], experiments of South Pole Telescope [22,23] and Atacama Cosmology Telescope [24], some authors obtained that the difference between the $\alpha$ today and at recombination was $\Delta \alpha / \alpha \leq 7.3 \times 10^{-3}$ at $68 \%$ of Confidence Level [25-34]. However, this limit obtained from the CMB physics is inferred considering a specific cosmological model (flat $\Lambda \mathrm{CDM}$ ), and being weakened by opening up the parameter space to variations of the number of relativistic species or the helium abundance. (see e.g. [35] and references therein).

A possible time variation of the fine structure constant during the Big Bang nucleosynthesis (BBN) [36] is also explored. Moreover, in the context of a supermassive black hole in the Galactic Center with a high gravitational poten- 
tial, it is used late-type evolved giant stars data from the S-star [37]. Recently, the Ref. [38] revisited the framework where the cosmological constant, $\Lambda$, is $\Lambda \propto \alpha^{-6}$ (the socalled $\Lambda(\alpha) C D M$ models). Using cosmological observations present in CAMB and CosmoMC packages and 313 data points from the absorption systems in the spectra of distant quasars, constraints on two specific $\Lambda(\alpha) \mathrm{CDM}$ models with one and two model parameters were performed. The authors found that the model parameters are constrained to be around $10^{-4}$, very similar to the results discussed by [39] but more accurately. However, the authors of the Ref. [40] showed that fitting turbulent models necessarily generate or enhance model non-uniqueness, adding a substantial additional random uncertainty to $\Delta \alpha / \alpha$.

Particularly, the low-energy string theory models predict the existence of a scalar field called dilaton, a spin- 2 graviton scalar partner $[17,41,42]$. In this scenario, the runaway of the dilaton towards strong coupling can lead to temporal variations of $\alpha$. However, the runaway dilaton and chameleon models have not been completely ruled out by the experiments that test violations on the weak equivalence principle [17,43-45]. Constraints on the Runaway Dilaton Model by using Galaxy clusters measurements have been proposed to probe a possible time variation in $\alpha$ (see the Ref. [46]). In [47], for instance, is introduced a method capable of probing a possible time variation in $\alpha$ by using galaxy cluster (GC) gas mass fraction measurements only. Constraints on $\Delta \alpha / \alpha$ achieved precision at the level $\sim 10^{-2}(1 \sigma$ c.l.). Using the angular diameter distance of GC and luminosity distance of type Ia supernovae, a possible temporal variation in $\alpha$ was also investigated, obtaining $\sim 10^{-2}$ at $1 \sigma$ c.l. [48]. Several other tests capable of probing $\alpha$ with galaxy cluster data have been emerging since then (see, for example, $[49,50]$ and references therein).

In this work, by assuming a flat universe, it is discussed for the first time the strong gravitational lensing (SGL) role on a possible temporal variation of the fine-structure constant. The proposed method is performed by using combined SGL systems and Type Ia Supernovae (SNe Ia). For that purpose, we use 92 pair of observations (SGL-SNe Ia) covering the redshift ranges $0.075 \leq z_{l} \leq 0.722$ and $0.2551 \leq z_{s} \leq 2.2649$. These data shall be considered to limit the $\gamma$ parameter, that is, considering runaway dilaton models. The approach developed here offers new limits on the $\gamma$ parameter using observations in higher redshifts than those from galaxy clusters $(z \approx 1)$.

This work is organized as follows: in Sect. 2 we shall discuss the theoretical model used to describe $\Delta \alpha / \alpha$. In Sect. 3 we describe the method developed to probe a time variation of $\alpha$. In Sect. 4 the data set to be used in our analyses, while Sect. 5 shows the results. Finally, in Sect. 6, the conclusions of this paper are presented.

\section{Theoretical framework}

In the modified gravity theories associated to a scalar field with non-minimal multiplicative coupling to the usual electromagnetic Lagrangian, the entire electromagnetic sector is changed (see details in [51,52]). Actually, such a nonminimal coupling is motivated by several alternative theories, as the low-energy action of string theories, in the context of axions, generalized chameleons, etc. In this kind of theory, a variation of $\alpha$ can arise either from a varying $\mu_{0}$ (vacuum permeability) or a variation of the charge of the elementary particles. Both interpretations lead to the same modified expression of the fine structure constant $[5,53,54]$.

In this paper, we focus on the runaway dilaton model $[41,42,51]$. The idea behind this model is to exploit the string-loop modifications of the four-dimensional effective low-energy action, where the Lagrangian is given by:

$\mathcal{L}=\frac{R}{16 \pi G}-\frac{1}{8 \pi G}(\nabla \phi)^{2}-\frac{1}{4} B_{F}(\phi) F^{2}+\cdots$,

here, $R$ is the Ricci scalar, $\phi$ is the scalar field named dilaton, $G$ is the gravitational constant, $F$ is the usual electromagnetic tensor, and $B_{F}$ is the gauge coupling function. From this action, the corresponding Friedmann equation and the motion equation for the dilaton field are given, respectively, by:

$$
H^{2}=8 \pi G \frac{\rho}{3+(1+z) \frac{d \phi}{d z}}
$$

and

$$
\begin{aligned}
& (1+z)^{2} \frac{d^{2} \phi}{d z^{2}}+\left[1-\frac{8 \pi G}{2 H^{2}}(\rho-p)\right](1+z) \frac{d \phi}{d z} \\
& =-\frac{8 \pi G}{2 H^{2}} \sum_{i} \beta_{i}(\phi)\left(\rho_{i}-3 p_{i}\right),
\end{aligned}
$$

where $H$ is the Hubble parameter concerning the components of the universe and dilaton field, the total energy density and the pressure are, respectively, $\rho=\sum_{i} \rho_{i}$ and $p=\sum_{i} p_{i}$, except the corresponding part of $\phi$. The $\beta_{i}$ are the couplings of $\phi$ with each component of matter $i$. However, the relevant parameter of the runaway dilaton model to study a possible time variation of $\alpha$ is the coupling of $\phi$ to the hadronic matter. The central hypothesis is that all gauge fields couple to the same $B_{F}$. From Eq. (1), it is possible one obtains $\alpha \propto B_{F}^{-1}(\phi)$ (see [46] and references therein). Thus, it follows:

$$
\frac{\Delta \alpha}{\alpha}=\frac{1}{40} \beta_{\text {had }, 0}\left[1-e^{-\left(\phi(z)-\phi_{0}\right)}\right],
$$

where $\beta_{\text {had }, 0}$ is the current value of the coupling between the dilaton and hadronic matter and

$$
\beta_{\text {had }}(\phi) \sim 40 \frac{\partial \ln B_{F}^{-1}(\phi)}{\partial \phi} \sim 1-b_{F} e^{-c \phi},
$$


where $c$ and $b_{F}$ are constant free parameters.

As we are interested in a possible time evolution of dilaton up to $z \approx 2.26$, an acceptable approximation to the field evolution is given by $\phi \sim \phi_{0}+\phi_{0}^{\prime} \ln a$, where $a$ is the cosmic scale factor [46]. Thus, one may obtain:

$\frac{\Delta \alpha}{\alpha} \approx-\frac{1}{40} \beta_{\text {had }, 0} \phi_{0}^{\prime} \ln (1+z) \approx-\gamma \ln (1+z)$,

where $\phi_{0}^{\prime} \equiv \frac{\partial \phi}{\partial \ln a}$ at the present time, and $\gamma \equiv \frac{1}{40} \beta_{\text {had }, 0} \phi_{0}^{\prime}$. This equation ${ }^{1}$ is that one we will use to compare the model predictions with combined SGL and SNe Ia data.

\section{Methodology}

Strong gravitational Lensing systems, one of the predictions of GR [55], have recently become a powerful astrophysical tool. They can investigate gravitational and cosmological theories, measure various cosmological parameters, and investigate fundamental physics. For example, time-delay measurements of gravitational lensings can be used to measure the Hubble constant [56], and the cosmic diameter distance relation (CDDR) [57]. Other statistical properties of SGL can restrict the deceleration parameter of the universe [58], spacetime curvature [59,60], also departures of CDDR [61,62], cosmological constant [63], the speed of light [64], and others. It is a purely gravitational phenomenon occurring when the source $(s)$, lens $(l)$, and observer $(o)$ are at the same signal line forming a structured ring called the Einstein radius $\left(\theta_{E}\right)$ [65]. In the cosmological scenario, a lens can be a foreground galaxy or cluster of galaxies positioned between a sourceQuasar, where the multiple-image separation from the source only depends on the lens and source angular diameter distances.

The system of SGL depends on a model for mass distribution. On the assumption of the singular isothermal sphere (SIS) model, the Einstein radius $\theta_{E}$ is given by [55]

$\theta_{E}=4 \pi \frac{D_{A_{l s}}}{D_{A_{s}}} \frac{\sigma_{S I S}^{2}}{c^{2}}$

where $D_{A_{l s}}$ is the angular diameter distance of the lens to the source, $D_{A_{s}}$ the angular diameter distance of the observer to the source, $c$ the speed of light, and $\sigma_{S I S}$ the velocity dispersion caused by the lens mass distribution. It is important to note here that $\sigma_{S I S}$ is not exactly equal to the observed stellar velocity dispersion $\left(\sigma_{0}\right)$ due to a strong indication, via X-ray observations, that dark matter halos are dynamically hotter

\footnotetext{
${ }^{1}$ As shown in the second panel of Fig.1 of the Ref. [46], the approach given by Eq. (6) can still be considered up to redshift $z \approx 5$ for values of the coupling that saturate the current bounds.
}

than luminous stars [66-69]. Taking this fact into account, we introduce a purely phenomenological free parameter: $f_{e}$, where $\sigma_{S I S}^{2}=\left(f_{e}\right)^{2} \sigma_{0}^{2}$, with $\sqrt{0.8}<f_{e}<\sqrt{1.2}$ (see [70]). As it is largely known, the $f_{e}$ parameter accounts not only for systematic errors caused by taking the observed stellar velocity dispersion as $\sigma_{S I S}$, but it also accounts for deviation of the real mass density profile from the SIS. Moreover, the effects of secondary lenses (mainly nearby galaxies) and line-of-sight contamination are also quantified by this factor (see also [71]).

The method developed by the Ref. [62] provided a robust test for CDDR using SGL systems and SNe Ia. The procedure is based on Eq. (7) for lenses and an observational quantity defined by

$D \equiv \frac{D_{A_{l s}}}{D_{A_{s}}}=\frac{\theta_{E} c_{S}^{2}}{4 \pi \sigma_{S I S}^{2}}$,

where $c_{s}$ is the speed of light measured between the source and us. However, such method did not take into consideration any possible variation of the fine structure constant on SGL observations. Here, we extend the method and investigate both effects of varying $\alpha$ and deviation of CDDR via SGL and SNe Ia observations. Thus, according to the definition of the fine structure constant $\left(\alpha_{s}=e^{2} / \hbar c_{s}\right)$ the Eq. (8) is rewritten by:

$D \equiv \frac{D_{A_{l s}}}{D_{A_{s}}}=\frac{e^{4} \theta_{E}}{\hbar^{2} \alpha_{S}^{2} 4 \pi \sigma_{S I S}^{2}}$.

On the other hand, assuming a flat universe with the comoving distance between the lens and the observer being $r_{l s}=r_{s}-r_{l}$, and using the relations $r_{s}=\left(1+z_{s}\right) D_{A_{s}}$, $r_{l}=\left(1+z_{l}\right) D_{A_{l}}, r_{l s}=\left(1+z_{s}\right) D_{A_{l s}}$, it is possible to obtain

$D=1-\frac{\left(1+z_{l}\right)}{\left(1+z_{S}\right)} \frac{D_{A_{l}}}{D_{A_{s}}}$.

Considering a possible deviation of CDDR by $D_{A_{i}}=$ $D_{L_{i}} / \eta\left(z_{i}\right) /\left(1+z_{i}\right)^{2}$, we obtain:

$D=1-\frac{\left(1+z_{s}\right) D_{L_{l}}}{\left(1+z_{l}\right) D_{L_{s}}} \frac{\eta\left(z_{s}\right)}{\eta\left(z_{l}\right)}$,

where $D_{L_{l}}$ and $D_{L_{s}}$ are the luminosity distances to lens and source, respectively, and $\eta\left(z_{i}\right)$ captures any deviation of CDDR.

As mentioned before, it was shown in Refs. [51,52] that for the class of theories obeying the Eq. (1), a variation of $\alpha$ necessarily leads to a violation of CDDR, and both changes are intimately and unequivocally related to each other by:

$\frac{\Delta \alpha}{\alpha} \equiv \frac{\alpha(z)-\alpha_{0}}{\alpha_{0}}=\eta^{2}(z)-1$. 
Considering $\alpha(z)=\alpha_{0} \phi(z)$, where $\alpha_{0}$ is the current value of the fine-structure constant, and $\phi(z)$ is a scalar field that controls a variation of $\alpha$, the Eq. (12) gives $\phi(z)=\eta^{2}(z)$. Thus, the Eqs. (9) and (11) shall be rewritten, respectively, by:

$D=\frac{e^{4} \theta_{E}}{4 \pi \alpha_{0}^{2} \hbar^{2} \sigma_{S I S}^{2}} \phi^{-2}\left(z_{s}\right)=D_{0} \phi^{-2}\left(z_{s}\right)$

and

$D=1-\frac{\left(1+z_{s}\right) D_{L_{l}}}{\left(1+z_{l}\right) D_{L_{s}}} \frac{\phi^{1 / 2}\left(z_{s}\right)}{\phi^{1 / 2}\left(z_{l}\right)}$,

where $D_{0} \equiv e^{4} \theta_{E} / 4 \pi \alpha_{0}^{2} \hbar^{2} \sigma_{S I S}^{2}$ (if $\Delta \alpha / \alpha=0$, so $\phi(z)=1$ and $D=D_{0}$ ). Therefore, combining Eqs. (13) and (14), it is possible to obtain

$D_{0}=\phi^{2}\left(z_{s}\right)\left[1-\frac{\left(1+z_{s}\right) D_{L_{l}}}{\left(1+z_{l}\right) D_{L_{s}}} \frac{\phi^{1 / 2}\left(z_{s}\right)}{\phi^{1 / 2}\left(z_{l}\right)}\right]$.

Note that if $\Delta \alpha / \alpha=0$, the quantity $D<1$, which means that systems with $D>1$ has no physical meaning.

\section{Samples}

\subsection{Type Ia Supernovae}

Now, let us consider the pair of luminosity distances for each SGL system, which we obtain from the SNe Ia sample called Pantheon [72]. It is worth mentioning that Pantheon is the most recent wide refined sample of $\mathrm{SNe}$ Ia observations found in the literature, consisting of 1049 spectroscopically confirmed SNe Ia and covers a redshift range of $0.01 \leq z \leq 2.3$. The sample of $D_{L}$ is constructed from the apparent magnitude $\left(m_{b}\right)$ of the Pantheon catalog by considering $M_{b}=-19.23 \pm 0.04$ (the absolute magnitude) by the relation

$D_{L}=10^{\left(m_{b}-M_{b}-25\right) / 5} \mathrm{Mpc}$.

However, to perform the appropriate tests, we must use $\mathrm{SNe}$ Ia at the same (or approximately) redshift of the lenssource of each system. Thus, we make a selection of $\mathrm{SNe}$ Ia according to the criterion: $\left|z_{s}-z_{S N e}\right| \leq 0.005$ and $\left|z_{l}-z_{S N e}\right| \leq 0.005$. Then, we perform the weighted average for each system by [62] (see Fig. 1):

$\bar{D}_{L}=\frac{\sum_{i} D_{L i} / \sigma_{D_{L i}}^{2}}{\sum_{i} 1 / \sigma_{D_{L i}}^{2}}$,

$\sigma_{\bar{D}_{L}}^{2}=\frac{1}{\sum_{i} 1 / \sigma_{D_{L i}}^{2}}$.

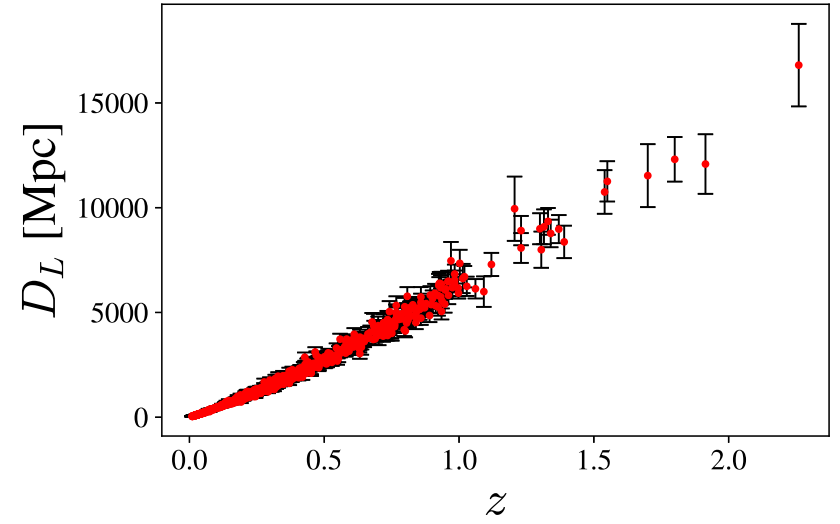

Fig. 1 Luminosity distances of spectroscopically confirmed SNe Ia from Pantheon compilation. Such a sample is constructed from the apparent magnitude $\left(m_{b}\right)$ of Pantheon catalog by considering $M_{b}=$ $-19.23 \pm 0.04$ (absolute magnitude)

It is important to stress that the influence of a possible variation of $\alpha$ on SNe Ia observations has been discussed in literature (see [73] and references therein). Briefly, the peak luminosities of $\mathrm{SNe}$ Ia depend on $\alpha$ and a variation of this constant directly translates into a different peak bolometric magnitude. In other words, the distance modulus is modified. However, the analyses of the Ref. [73] concluded that at $3 \sigma$, the parameters of the SNe Ia data used (JLA and Union2.1 compilations) are consistent with a null variation of $\alpha$.

\subsection{SGL systems}

We consider a specific catalog containing 158 confirmed sources of strong gravitational lensing by [74]. This compilation includes 118 SGL systems identical to the compilation of [55]. The SGL were obtained from SLOAN Lens ACS, BOSS Emission-line Lens Survey (BELLS), and Strong Legacy Survey SL2S, along with 40 new systems recently discovered by SLACS and pre-selected by [75] (see Table I in [74]).

However, studies using lensing systems have shown that the pure SIS model may not be an accurate representation of the lens mass distribution when $\sigma_{0}<250 \mathrm{~km} / \mathrm{s}$, for which non-physical values of the quantity $D_{0}$ are usually found $\left(D_{0}>1\right)$. In [74] is also mentioned the need for attention when the SIS model is used as a reference since the impact caused on the density profile can cause deviations on the observed stellar velocity dispersion $\left(\sigma_{0}\right)$. For this reason, by excluding non-physical measurements of $D_{0}$, and the system J0850-0347 ${ }^{2}$ [74], our sample finishes with 140 measurements of $D_{0}$.

We also consider a general approach to describe the lensing systems: the one with spherically symmetric mass distribution in lensing galaxies in favor of power-law index $\Upsilon$,

\footnotetext{
${ }^{2}$ It deviates by more than $5 \sigma$ from all the considered models.
} 
where $\rho \propto r^{-\Upsilon}$ (PLAW). This kind of model is essential since several recent studies have shown that slopes of density profiles of individual galaxies show a non-negligible scatter from the SIS model [66-69]. Under this assumption, the quantity $D_{0}$ of Eq. (13) shall be rewritten by:

$D_{0}=\frac{e^{4} \theta_{E}}{\alpha_{0}^{2} \hbar^{2} 4 \pi \sigma_{a p}^{2}}\left(\frac{\theta_{a p}}{\theta_{E}}\right)^{2-\Upsilon} f^{-1}(\Upsilon)$,

where $\sigma_{a p}$ is stellar velocity dispersion inside an aperture of size $\theta_{a p}, \Upsilon$ the power-law index (if $\Upsilon=2$, Eq. (19) resumes the SIS model), and

$f(\Upsilon)=-\frac{(5-2 \Upsilon)(1-\Upsilon)}{\sqrt{\pi}(3-\Upsilon)} \frac{\Gamma(\Upsilon-1)}{\Gamma(\Upsilon-3 / 2)}\left[\frac{\Gamma(\Upsilon / 2-1 / 2)}{\Gamma(\Upsilon / 2)}\right]^{2}$.

In this paper, the factor $\Upsilon$ is approached as a free parameter. ${ }^{3}$ The uncertainty related to Eq. (19) is given by:

$\sigma_{D_{0}}=D_{0} \sqrt{4\left(\frac{\sigma_{\sigma_{a p}}}{\sigma_{a p}}\right)^{2}+(1-\Upsilon)^{2}\left(\frac{\sigma_{\theta_{E}}}{\theta_{E}}\right)^{2}}$.

Following the approach taken by [79], Einstein's radius uncertainties follows $\sigma_{\theta_{E}}=0.05 \theta_{E}$ ( $5 \%$ for all systems).

As mentioned before, our sample consists of $140 \mathrm{SGL}$ systems covering a wide range of redshift. However, not all the SGL systems have the corresponding pair of luminosity distances via $\mathrm{SNe}$ Ia that obey the previous criteria. We ended up with 92 pairs of observations (SGL-SNe Ia) for our analyses by also excluding these systems.

\section{Analysis and discussions}

We used Markov Chain Monte Carlo (MCMC) methods to calculate the posterior probability distribution functions (pdf) of free parameters [80]. For SIS model, the free parameter space is $\vec{\Theta}=\left(\gamma, f_{e}\right)$, and for PLAW model is $\vec{\Theta}=(\gamma, \Upsilon)$. Thus, the likelihood distribution function is given by:

$\mathcal{L}($ Data $\mid \vec{\Theta})=\prod \frac{1}{\sqrt{2 \pi} \sigma_{\mu}} \exp \left(-\frac{1}{2} \chi^{2}\right)$,

where

$$
\begin{aligned}
\chi^{2} & =\left[\frac{\left(D_{0}-\zeta\right)}{\sigma_{T}}\right]^{2}, \\
\zeta & \equiv \phi^{2}\left(z_{s}\right)\left[1-\frac{\left(1+z_{s}\right) D_{L_{l}}}{\left(1+z_{l}\right) D_{L_{s}}} \frac{\phi^{1 / 2}\left(z_{s}\right)}{\phi^{1 / 2}\left(z_{l}\right)}\right],
\end{aligned}
$$

\footnotetext{
3 This method is widely used in the literature [76-78].
}

$$
\begin{aligned}
\sigma_{T} & =\left(\sigma_{D_{0}}^{2}+\sigma_{\zeta}^{2}\right)^{1 / 2}, \\
\sigma_{\zeta}^{2} & =\frac{\phi^{5}\left(z_{s}\right)}{\phi\left(z_{l}\right)}\left[\frac{\left(1+z_{s}\right)}{\left(1+z_{l}\right)} \frac{D_{L_{l}}}{D_{L_{s}}}\right]^{2} \cdot K, \\
K & \equiv\left\{\left(\frac{\sigma_{D_{L_{l}}}}{D_{L_{l}}}\right)^{2}+\left(\frac{\sigma_{D_{L_{s}}}}{D_{L_{s}}}\right)^{2}\right\}
\end{aligned}
$$

the associated errors. As mentioned before, $\phi\left(z_{s}\right)=1-$ $\gamma \ln \left(1+z_{s}\right)$ and $\phi\left(z_{l}\right)=1-\gamma \ln \left(1+z_{l}\right)$, where $\gamma$ is the parameter to be constrained. The pdf posteriori is proportional to the product between the likelihood and the prior, that is,

$P(\vec{\Theta} \mid$ Data $) \propto \mathcal{L}($ Data $\mid \vec{\Theta}) \times P_{0}(\vec{\Theta})$.

In our analysis, we assume flat priors: $-1.0 \leq \gamma \leq+1.0$; $1.5 \leq \Upsilon \leq 2.5 ; \sqrt{0.8} \leq f_{e} \leq \sqrt{1.2}$.

Our main results are :

- For the SIS model: $\gamma=0.04_{-0.06}^{+0.05}$, and $f_{e}=1.02_{-0.02}^{+0.02}$, where $\chi_{\text {red }}^{2} \approx 1.57(1 \sigma)$.

- For the PLAW model: $\gamma=-0.03_{-0.02}^{+0.02}$, and $\Upsilon=$ $1.99_{-0.04}^{+0.04}(1 \sigma)$, where $\chi_{\text {red }}^{2} \approx 1.58(1 \sigma)$.

As one may see, the PLAW model agrees to the SIS model within $2 \sigma$ c.l..

As mentioned by [74], it is necessary to add $12.22 \%$ of intrinsic error associated to $D_{0}$ measurement. As the random variation in galaxy morphology is almost Gaussian, the authors of Ref. [74] found that an additional error term of about $12.22 \%$ is necessary to have $68.3 \%$ of the observations lie within $1 \sigma$ of the best-fit $\omega \mathrm{CDM}$ model, which is smaller than $20 \%$ scatter as suggested by [55]. Moreover, this procedure makes $D$ more homogeneous for the lensing sample located at different redshifts. Therefore, our main results are:

- For the SIS model: $\gamma=0.04_{-0.08}^{+0.07}$ and $f_{e}=1.02_{-0.03}^{+0.03}$, where $\chi_{\text {red }}^{2} \approx 0.91$ (see Fig. 2).

- For the PLAW model: $\gamma=-0.03_{-0.04}^{+0.03}$ and $\Upsilon=$ $1.97_{-0.05}^{+0.05}$, where $\chi_{\text {red }}^{2} \approx 0.91$ (see Fig. 3).

Table 1 shows the bounds on $\gamma$ derived in this paper, along with other recent constraints obtained from galaxy clusters and $\mathrm{SNe}$ Ia observations. As one may see, our results are in full agreement with the previous ones from galaxy clusters plus SNe Ia analyses.

\section{Conclusions}

The search for a possible temporal or/and spatial variation of the fundamental constants of nature has received significant 


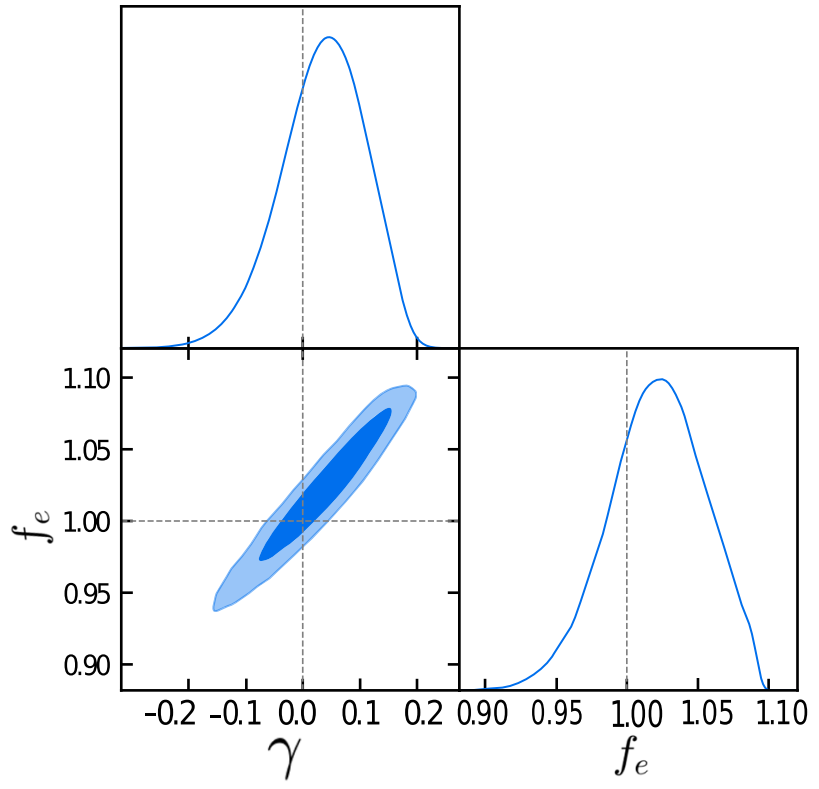

Fig. 2 Posteriori probability distribution of free parameters $\gamma$ and $f_{e}$ for SIS model considering $\sigma_{i n t} \approx 12.22 \%$. The vertical dashed lines correspond to no variation of $\alpha$ and $\sigma_{S I S}=\sigma_{0}$

interest in the last decades, given the improvement in astrophysics' observational data. In this paper, a new technique was proposed to investigate a possible time variation of the fine structure constant, such as $\alpha(z)=\alpha_{0} \phi(z)$, with data at high redshifts by using recent measurements of SGL systems and SNe Ia observations. A possible time variation of $\alpha$ in a class of runaway dilaton models, with $\phi(z)=1-\gamma \ln (1+z)$, was investigated.

As we have already discussed, considering the SIS model to describe the mass distribution in lensing galaxies, we obtained: $\gamma=+0.04_{-0.06}^{+0.05}$ and $f_{e}=1.02_{-0.02}^{+0.02}$. By considering the PLAW model, we obtained: $\gamma=-0.03_{-0.02}^{+0.02}$ and $\Upsilon=1.99_{-0.04}^{+0.04}$. By adding $\sigma_{\text {int }} \approx 12.22 \%$ of intrinsic error, we obtain: for the SIS model $\gamma=0.04_{-0.08}^{+0.07}$ and

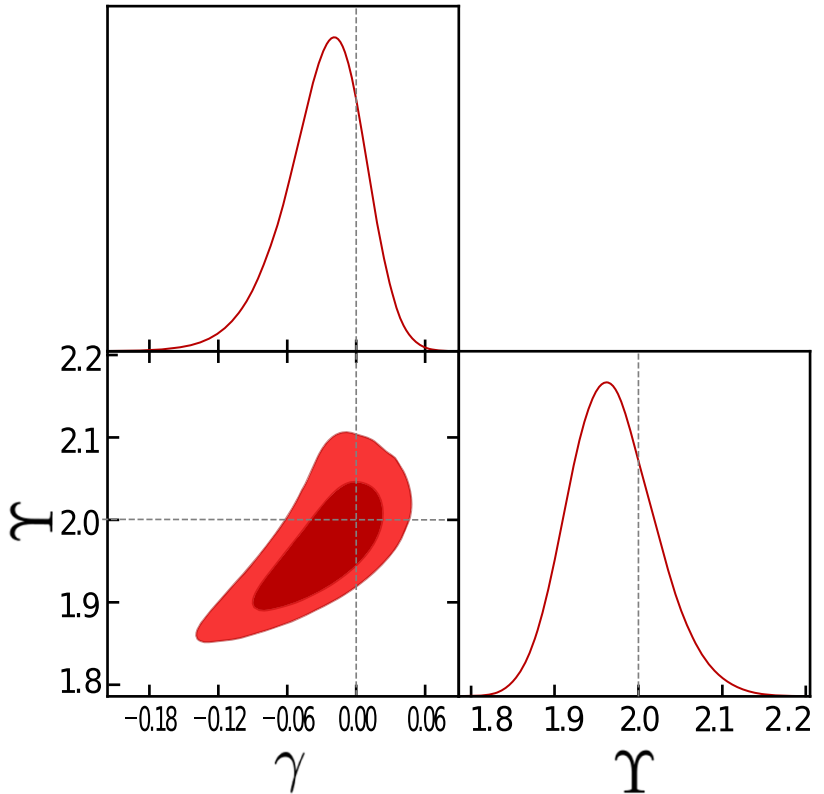

Fig. 3 Posteriori probability distribution of free parameters $\gamma$ and $\Upsilon$ for the PLAW model considering $\sigma_{i n t} \approx 12.22 \%$. The vertical dashed lines correspond to no variation of $\alpha$. As one may see, the PLAW model agrees to the SIS model within $1 \sigma$ c.l.

$f_{e}=1.02_{-0.03}^{+0.03}$, and for the PLAW model $\gamma=-0.03_{-0.04}^{+0.03}$ and $\Upsilon=1.97_{-0.05}^{+0.05}$, both in $1 \sigma$ of confidence level. These results are in full agreement with the standard cosmology. Although SGL systems data are not competitive with the limits imposed by quasar absorption systems, the constraints imposed in this paper provide new and independent limits on a possible time variation of the fine structure constant.

Finally, as an interesting extension of the present work, one may check the consequences of relaxing the rigid assumption that the stellar luminosity and total mass distributions follow the same power law $[82,83]$. Moreover, the well-known Mass-sheet degeneracy (see [84] and references

Table 1 A summary of current constraints on a possible time evolution of $\alpha$ for a class of runaway dilaton models $(\Delta \alpha / \alpha=-\gamma \ln (1+z))$ by using galaxy cluster observations and SNe Ia measurements. The symbol * denotes galaxy cluster data

\begin{tabular}{lll}
\hline Data set & Profile & $\gamma$ \\
\hline Gas Mass Fractions* [47] & Non-Isothermal double $\beta$-Model & $+0.065 \pm 0.095$ \\
Angular Diameter Distance* plus SNe Ia [48] & Isothermal Elliptical $\beta$-Model & $-0.037 \pm 0.157$ \\
Gas Mass Fractions* plus SNe Ia [49] & Universal Pressure Profile & $+0.008 \pm 0.035$ \\
Gas Mass Fractions* plus SNe Ia [49] & Virialized ideal gas & $+0.018 \pm 0.032$ \\
Gas Mass Fractions* plus SNe Ia [49] & Non-thermal Pressure and Adiabatic Model & $+0.010 \pm 0.030$ \\
Gas Mass Fractions* plus SNe Ia [49] & Mass Dynamical Estimate from Galaxy Velocity Dispersions & $+0.030 \pm 0.033$ \\
$Y_{S Z} D_{A}^{2} / Y_{X}$ scaling-relation* [81] & Universal pressure profile & $-0.15 \pm 0.10$ \\
This work-SGL & SIS Model & $+0.04_{-0.08}^{+0.07}$ \\
This work-SGL & PLAW Model & $-0.03_{-0.04}^{+0.03}$ \\
\hline
\end{tabular}


therein) in the gravitational lens system and its effect on our results also could be explored further.

Acknowledgements The authors thank Brazilian scientific and financial support federal agencies, CAPES, and CNPq. RS thanks CNPq (Grant No. 307620/2019-0) for financial support. This work was supported by the High-Performance Computing Center (NPAD)/UFRN.

Data Availability Statement This manuscript has no associated data or the data will not be deposited. [Authors' comment: The data sets we used in our analyses are available in the cited articles.]

Open Access This article is licensed under a Creative Commons Attribution 4.0 International License, which permits use, sharing, adaptation, distribution and reproduction in any medium or format, as long as you give appropriate credit to the original author(s) and the source, provide a link to the Creative Commons licence, and indicate if changes were made. The images or other third party material in this article are included in the article's Creative Commons licence, unless indicated otherwise in a credit line to the material. If material is not included in the article's Creative Commons licence and your intended use is not permitted by statutory regulation or exceeds the permitted use, you will need to obtain permission directly from the copyright holder. To view a copy of this licence, visit http://creativecomm ons.org/licenses/by/4.0/.

Funded by SCOAP ${ }^{3}$.

\section{References}

1. P.A.M. Dirac, The cosmological constants. Nature 139, 323 (1937)

2. E. Teller, On the change of physical constants. Phys. Rev. 73, 801 (1948)

3. E.P. Bellinger, J. Christensen-Dalsgaard, Asteroseismic constraints on the cosmic-time variation of the gravitational constant from an ancient main-sequence star. Astrophys. J. 887, 1 (2019). arXiv: 1909.06378

4. J. Muller, L. Biskupek, Variations of the gravitational constant from lunar laser ranging data. Class. Quantum Gravity 24, 4533 (2007). arXiv:gr-qc/0509114

5. J.P. Uzan, Varying constants, gravitation and cosmology. Living Rev. Relativ. 14, 2 (2011). arXiv: 1009.5514

6. T. Chiba, The constancy of the constants of nature: updates. Prog. Theor. Phys. 126, 993 (2011). arXiv: 1111.0092

7. A. Chodos, S.L. Detweiler, Where has the fifth-dimension gone? Phys. Rev. D 21, 2167 (1980)

8. E.W. Kolb, M.J. Perry, T.P. Walker, Time variation of fundamental constants, primordial nucleosynthesis and the size of extra dimensions. Phys. Rev. D 33, 869 (1986)

9. P. Nath, M. Yamaguchi, Effects of extra space-time dimensions on the Fermi constant. Phys. Rev. D 60, 116004 (1999)

10. J.D. Bekenstein, Fine structure constant: is it really a constant? Phys. Rev. D 25, 1527 (1982)

11. J.K. Webb, J.A. King, M.T. Murphy, V.V. Flambaum, R.F. Carswell, M.B. Bainbridge, Indications of a spatial variation of the fine structure constant. Phys. Rev. Lett. 107, 191101 (2011). arXiv: 1008.3907

12. J.A. King, J.K. Webb, M.T. Murphy, V.V. Flambaum, R.F. Carswell, M.B. Bainbridge, M.R. Wilczynska, F.E. Koch, Spatial variation in the fine-structure constant-new results from VLT/UVES. Mon. Not. R. Astron. Soc. 422, 3370 (2012). arXiv: 1202.4758

13. J.K. Webb, V.V. Flambaum, C.W. Churchill, M.J. Drinkwater, J.D. Barrow, A search for time variation of the fine structure constant. Phys. Rev. Lett. 82, 884 (1999). arXiv:astro-ph/9803165
14. M.T. Murphy, J.K. Webb, V.V. Flambaum, Further evidence for a variable fine-structure constant from Keck/HIRES QSO absorption spectra. Mon. Not. R. Astron. Soc. 345, 609 (2003). arXiv:astro-ph/0306483

15. A. Songaila, L.L. Cowie, Constraining the variation of the fine structure constant with observations of narrow quasar absorption lines. Astrophys. J. 793, 103 (2014). arXiv:1406.3628

16. C.J.A.P. Martins, A.M.M. Pinho, Stability of fundamental couplings: a global analysis. Phys. Rev. D 95, 023008 (2017). arXiv: 1701.08724

17. C.J.A.P. Martins, The status of varying constants: a review of the physics, searches and implications. arXiv:1709.02923

18. T. Damour, F. Dyson, The Oklo bound on the time variation of the fine structure constant revisited. Nucl. Phys. B 480, 37 (1996). arXiv:hep-ph/9606486

19. M.R. Wilczynska et al., Four direct measurements of the finestructure constant 13 billion years ago (2020). arXiv:2003.07627

20. P.A.R. Ade et al. [Planck Collaboration], Planck 2015 results. XIII. Cosmological parameters. Astron. Astrophys. 594, A13 (2016). arXiv: 1502.01589

21. N. Aghanim et al. [Planck Collaboration], "Planck 2018 results. VI. Cosmological parameters", [arXiv:1807.06209]

22. K.T. Story et al., A measurement of the cosmic microwave background damping tail from the 2500-square-degree SPT-SZ survey. Astrophys. J. 779, 86 (2013). arXiv:1210.7231

23. B.A. Benson et al., [SPT-3G Collaboration], SPT-3G: a nextgeneration cosmic microwave background polarization experiment on the south pole telescope. Proc. SPIE Int. Soc. Opt. Eng. 9153, 91531P (2014). arXiv:1407.2973

24. T. Louis et al. [ACTPol Collaboration], The Atacama Cosmology Telescope: two-season ACTPol spectra and parameters. J. Cosmol. AP 1706, 031 (2017). arXiv: 1610.02360

25. P.P. Avelino et al., Early universe constraints on a time varying fine structure constant. Phys. Rev. D 64, 103505 (2001). arXiv:astro-ph/0102144

26. C.J.A.P. Martins, A. Melchiorri, G. Rocha, R. Trotta, P.P. Avelino, P.T.P. Viana, Wmap constraints on varying alpha and the promise of reionization. Phys. Lett. B 585, 29 (2004). arXiv:astro-ph/0302295

27. G. Rocha, R. Trotta, C.J.A.P. Martins, A. Melchiorri, P.P. Avelino, R. Bean, P.T.P. Viana, Measuring alpha in the early universe: $\mathrm{cmb}$ polarization, reionization and the fisher matrix analysis. Mon. Not. R. Astron. Soc. 352, 20 (2004). arXiv:astro-ph/0309211

28. K. Ichikawa, T. Kanzaki, M. Kawasaki, CMB constraints on the simultaneous variation of the fine structure constant and electron mass. Phys. Rev. D 74, 023515 (2006). arXiv:astro-ph/0602577

29. E. Menegoni, S. Galli, J.G. Bartlett, C.J.A.P. Martins, A. Melchiorri, New constraints on variations of the fine structure constant from CMB anisotropies. Phys. Rev. D 80, 087302 (2009). arXiv:0909.3584

30. S. Galli, M. Martinelli, A. Melchiorri, L. Pagano, B.D. Sherwin, D.N. Spergel, Constraining fundamental physics with future CMB experiments. Phys. Rev. D 82, 123504 (2010). arXiv:1005.3808

31. E. Menegoni, M. Archidiacono, E. Calabrese, S. Galli, C.J.A.P. Martins, A. Melchiorri, The fine structure constant and the CMB damping scale. Phys. Rev. D 85, 107301 (2012). arXiv:1202.1476

32. P.A.R. Ade et al. [Planck Collaboration], Planck intermediate results-XXIV. Constraints on variations in fundamental constants. Astron. Astrophys. 580, A22 (2015). arXiv:1406.7482

33. I. de Martino, C.J.A.P. Martins, H. Ebeling, D. Kocevski, Constraining spatial variations of the fine structure constant using clusters of galaxies and Planck data. Phys. Rev. D 94, 083008 (2016). arXiv: 1605.03053

34. L. Hart, J. Chluba, New constraints on time-dependent variations of fundamental constants using Planck data. Mon. Not. R. Astron. Soc. 474, 1850 (2018). arXiv:1705.03925 
35. T.L. Smith, D. Grin, D. Robinson, D. Qi, Probing spatial variation of the fine-structure constant using the CMB. Phys. Rev. D 99, 043531 (2019). arXiv: 1808.07486

36. M.E. Mosquera, O. Civitarese, Chameleon fields: awaiting surprises for tests of gravity in space. Astron. Astrophys. 551, A122 (2013). arXiv:astro-ph/0309300

37. A. Hees, T. Do, B.M. Roberts, A.M. Ghez, S. Nishiyama, R.O. Bentley, A.K. Gautam, S. Jia, T. Kara, J.R. Lu, H. Saida, S. Sakai, M. Takahashi, Y. Takamori, Search for a variation of the fine structure around the supermassive black hole in our galactic center. Phys. Rev. Lett. 124, 081101 (2020). arXiv:astro-ph/2002.11567

38. J.-J. Zhang, L. Yin, C.-Q. Geng, Cosmological constraints on $\Lambda(\alpha)$ CDM models with time-varying fine structure constant. Ann. Phys. 397, 400-409 (2018). arXiv:1809.04218

39. H. Wein, X.-B. Zou, H.Y. Li, D.Z. Xue, Cosmological constant, fine structure constant and beyond. Eur. Phys. J. C 77, 1 (2017). arXiv: 1605.04571

40. C.-C. Lee, J.K. Webb, D. Milaković, R.F. Carswell, Nonuniqueness in quasar absorption models and implications for measurements of the fine-structure constant (2021). arXiv:2102.11648

41. T. Damour, F. Piazza, G. Veneziano, Violations of the equivalence principle in a dilaton-runaway scenario. Phys. Rev. D 66, 4 (2002). arXiv:hep-th/0205111v2

42. T. Damour, F. Piazza, G. Veneziano, Runaway dilaton and equivalence principle violations. Phys. Rev. Lett. 89, 8 (2002). arXiv:gr-qc/0204094v2

43. J. Khoury, A. Weltman, Chameleon fields: awaiting surprises for tests of gravity in space. Phys. Rev. Lett. 93, 171104 (2004). arXiv:astro-ph/0309300

44. P. Brax, C. van de Bruck, A.-C. Davis, J. Khoury, A. Weltman, Detecting dark energy in orbit: the cosmological chameleon. Phys. Rev. D 70, 123518 (2004). arXiv:astro-ph/0408415

45. D.F. Mota, D.J. Shaw, Evading equivalence principle violations, astrophysical and cosmological constraints in scalar field theories with a strong coupling to matter. Phys. Rev. D 75, 063501 (2007). arXiv:hep-ph/0608078

46. C.J.A.P. Martins, P.E. Vielzeuf, M. Martinelli, E. Calabrese, S. Pandolfi, Evolution of the fine-structure constant in runaway dilaton models. Phys. Lett. B 743, 377-382 (2015). arXiv:1503.05068

47. R.F.L. Holanda, S.J. Landau, J.S. Alcaniz, I.E. Sanchez, V.C. Busti, Constraints on a possible variation of the fine structure constant from galaxy cluster data. J. Cosmol. Astropart. Phys. 1605, 047 (2016). arXiv: 1510.07240

48. R.F.L. Holanda, V.C. Busti, L.R. Colaço, J.S. Alcaniz, S.J. Landau, Galaxy clusters, type Ia supernovae and the fine structure constant. J. Cosmol. Astropart. Phys. 1608, 055 (2016). arXiv:1605.02578

49. R.F.L. Holanda, L.R. Colaço, R.S. Gonçalves, J.S. Alcaniz, Limits on evolution of the fine-structure constant in runaway dilaton models from Sunyaev-Zeldovich Observations. Phys. Lett. B 767, 188-192 (2017). arXiv: 1701.07250

50. I. de Martino, C.J.A.P. Martins, H. Ebeling, D. Kocevski, New constraints on spatial variations of the fine structure constant from clusters of galaxies. Phys. Rev. D 2, 034 (2016). arXiv:1612.06739v1

51. O. Hees, A. Minazzoli, J. Larena, Breaking of the equivalence principle in the electromagnetic sector and its cosmological signatures. Phys. Rev. D 90, 12 (2014). arXiv:1406.6187v4

52. O. Minazzoli, A. Hees, Late-time cosmology of a scalar-tensor theory with a universal multiplicative coupling between the scalar field and the matter Lagrangian. Phys. Rev. D 90, 2 (2014). arXiv:1404.4266v2

53. A. Hess, O. Minazzoli, J. Larena, Observables in theories with a varying fine structure constant. Gen. Relativ. Gravit. 47, 2 (2015). arXiv: 1409.7273

54. J.D. Bekenstein, Fine-structure constant: is it really a constant? PRD 25, 6 (1982)
55. S. Cao, M. Biesiada, R. Gavazzi, A. Piórkowska, Z.-H. Zhu, Cosmology with strong-lensing systems. Astrophy. J. 806, 185 (2015). arXiv: 1509.07649

56. C.S. Kochanek, P.L. Schechter, The Hubble Constant from Gravitational Lens time Delays, Measuring and Modeling the Universe", from the Carnegie Observatories Centennial Symposia. Published by Cambridge University Press, as part of the Carnegie Observatories Astrophysics Series, ed. by W.L. Freedman (2004), p. 117. arXiv:astro-ph/0306040

57. A. Rana, D. Jain, S. Mahajan, A. Muherjee, R.F.L. Holanda, Probing the cosmic distance duality relation using time delay lenses. J. Cosmol. Astropart. Phys. 1707, 010 (2017). arXiv:1705.04549

58. J.R. Gott, M.-G. Park, H.M. Lee, Settings limits on $q_{0}$ from gravitational lensing. Astrophys. J. 338, 1-12 (1989)

59. J.-Z. Qi, S. Cao, S. Zhang, M. Biesiada, Y. Wu, Z.-H. Zhu, The distance sum rule from strong lensing systems and quasars-test of cosmic curvatura and beyond. Mon. Not. R. Astron. Soc. 483, 1 (2019). arXiv: 1803.01990

60. A. Rana, D. Jain, S. Mahajan, A. Mukherjee, Constraining cosmic curvature by using age of galaxies and gravitational lenses. J. Cosmol. Astropart. Phys. 028, 03 (2017). arXiv:1611.07196

61. C.-Z. Ruan, F. Melia, T.-J. Zhang, Model-independent test of the cosmic distance duality relation. Astrophys. J. 866, 31 (2018). arXiv: 1808.09331

62. R.F.L. Holanda, V.C. Busti, F.S. Lima, J.S. Alcaniz, Probing the distance-duality relation with high-z data. J. Cosmol. Astropart. Phys. 1709, 039 (2017). arXiv: 1611.09426

63. M. Fukugita, T. Futamase, M. Kasa, E.L. Turner, Statistical properties of gravitational lenses with a nonzero cosmological constant. Astrophys. J. 393, 1 (1992)

64. S. Cao, J. Qi, M. Biesiada, X. Zheng, T. Xu, Z.-H. Zhu, Testing the speed of the light over cosmological distances: the combination of strongly lensed and unlensed supernova Ia. Astrophys. J. 867, 50 (2018). arXiv: 1810.01287

65. P. Schneiner, J. Ehlers, E.E. Falco, Gravitational Lendes, Springer, Berlin. Also Astronomy and Astrophysics Library (2019)

66. L. Koopmans, A. Bolton, T. Treu, O. Czoske, M. Auger et al., The structure and dynamics of massive early-type galaxies: on homology, isothermality, and isotropy inside one effective radius. Astrophys. J. 703, L54 (2009). arXiv:0906.1349

67. M.W. Auger, T. Treu, A.S. Bolton, R. Gavazzi, L.V.E. Koopmans, P.J. Marshall, L.A. Moustakas, S. Burles, The Sloan Lens ACS Survey. X. Stellar, dynamical, and total mass correlations of massive early-type galaxies. Astrophys. J. 724, 511 (2010). arXiv: 1007.2880

68. M. Barnabe, O. Czoske, L.V.E. Koopmans, T. Treu, A.S. Bolton, Two-dimensional kinematics of SLACS lenses-III. Mass structure and dynamics of early-type lens galaxies beyond $z \approx 0.1$. Mon. Not. R. Astron. Soc. 415, 2215 (2011). arXiv:1102.2261

69. A. Sonnenfeld, T. Treu, R. Gavazzi, S.H. Suyu, P.J. Marshall et al., The SL2S Galaxy-Scale Lens Sample. IV. The dependence of the total mass density profile of early-type galaxies on redshift, stellar mass, and size. Astrophys. J. 777, 98 (2013). arXiv:1307.4759

70. S. Cao, Y. Pan, M. Biesiada, W. Godlowski, Z.-H. Zhu, Constraints on cosmological models from strong gravitational lensing systems. JCAP 2012, 3 (2012). arXiv:1105.6226

71. E.O. Ofek, H.-W. Rix, D. Maoz, The redshift distribution of gravitational lenses revisited: constraints on galaxy mass evolution. Mon. Not. R. Astron. Soc. 343, 639 (2003). arXiv:astro-ph/0305201v1

72. D.M. Scolnic et al., The complete Ligh-curve sample of spectroscopically confirmed SNe Ia from Pan-STARRS1 and cosmological constraints from the combined pantheon sample. Astrophys. J. 859, 101 (2018). arXiv: 1710.00845

73. L. Kraiselburd, S. Landau, E. García-Berro, Spatial variation of fundamental constants: testing models with thermonuclear supernovae. Int. J. Mod. Phys. D 27, 1850099 (2018) 
74. K. Leaf, F. Melia, Model selection with strong-lensing systems. MNRAS 478, 4 (2018). arXiv: 1805.08640

75. Y. Shu, J.R. Brownstein, A.S. Bolton, L.V.E. Koopmans, T. Treu, A.D. Montero-Dorta, M.W. Auger, O. Czoske, R. Gavazzi, P.J. Marshall, L.A. Moustakas, The Sloan Lens ACS Survey. XIII. Discovery of 40 new galaxy-scale strong lenses. ApJ 851, 1 (2017). arXiv: 1711.00072

76. J.-Q. Xia, H. Yu, G.-J. Wang, S.-X. Tian, Z.-X. Li, S. Cao, Z.-H. Zhu, Revesting studies of the statistical property of a strong gravitational lens system and model-independent constraint on the curvature of the universe. Astrophys. J. 834, 1 (2017). arXiv:1611.04731

77. Z. Li, X. Ding, G.-J. Wang, K. Liao, Z.-H. Zhu, Curvature from strong gravitational lensing: a spatially closed universe or systematics? Astrophys. J. 854, 146 (2018). arXiv:1801.08001

78. X. Li, L. Tang, H.-N. Lin, Probing cosmic acceleration by strong gravitational lensing systems. Mon. Not. R. Astron. Soc. 484, 3 (2019). arXiv:1901.09144v1

79. C. Grillo, M. Lombardi, G. Bertin, Cosmological parameters from strong gravitational lensing and stellar dynamics in elliptical galaxies. Astron. Astrophys. 477, 397 (2008). arXiv:0711.0882
80. D. Foreman-Mackey, D.W. Hogg, D. Lang, J. Goodman, emcee: the MCMC Hammer. Publ. Astron. Soc. Pac. 125, 925 (2013). arXiv: 1202.3665

81. L.R. Colaço, R.F.L. Holanda, R. Silva, J.S. Alcaniz, Galaxy clusters and a possible variation of the fine structure constant. JCAP 03, 014 (2019). arXiv: 1901.10947

82. S. Cao, M. Biesiada, X. Zheng, Z.-H. Zhu, Testing the gas mass density profile of galaxy clusters with distance duality relation. Mon. Not. R. Astron. Soc. 457, 1 (2016). arXiv:1601.00409

83. J. Schwab, A.S. Bolton, S.A. Rappaport, Galaxy-scale strong lensing tests of gravity and geometric cosmology: constraints and systematic limitations. Astrophys. J. 708, 750-757 (2010). arXiv:0907.4992

84. S. Birrer, A. Amara, A. Refregier, The mass-sheet degeneracy and time-delay cosmography: analysis of the strong lens RXJ11311231. JCAP 08, 020 (2016). arXiv: 1511.03662 